\title{
Monitoring Social Distancing in Smart Spaces using Infrastructure-Based Sensors
}

\section{Hossein Motlagh, Naser}

\section{IEEE}

2021-11-09

Hossein Motlagh , N , Toivonen , P , Zaidan , M A , Lagerspetz , E, Peltonen , E , Gilman , E , Nurmi , P \& Tarkoma, S 2021, Monitoring Social Distancing in Smart Spaces using Infrastructure-Based Sensors . in IEEE 7th World Forum on Internet of Things (WF-IoT 2021) . IEEE , pp. 124-129, IEEE World Forum on Internet of Things, New Orleans, United States , 14/06/2021 . https://doi.org/10.1109/WF-loT51360.2021.9595897

http://hdl.handle.net/10138/336412

https://doi.org/10.1109/WF-loT51360.2021.9595897

unspecified

acceptedVersion

Downloaded from Helda, University of Helsinki institutional repository.

This is an electronic reprint of the original article.

This reprint may differ from the original in pagination and typographic detail.

Please cite the original version. 


\title{
Monitoring Social Distancing in Smart Spaces using Infrastructure-Based Sensors
}

\author{
Naser Hossein Motlagh $\dagger$, Pupu Toivonen $\dagger$, Martha Arbayani Zaidan $\S^{*}$, Eemil Lagerspetz $\dagger$,

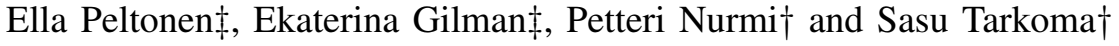 \\ $\dagger$ Department of Computer Science, University of Helsinki, Finland \\ $\S$ Institute for Atmospheric and Earth System Research (INAR), University of Helsinki, Finland \\ * Joint International Research Laboratory of Atmospheric and Earth System Sciences, Nanjing University, China \\ $\ddagger$ Center for Ubiquitous Computing, University of Oulu, Oulu, Finland \\ Emails: †£ firstname.lastname@ @elsinki.fi, łfirstname.lastname@oulu.fi
}

\begin{abstract}
Social distancing is a critical tool for mitigating disease transmission, particularly in crowded indoor spaces. In this paper, we contribute by assessing the feasibility of re-purposing existing infrastructure of occupancy monitoring sensors and environmental sensors for the dual purpose of monitoring social distancing and supporting disease transmission risk estimation. We consider 410 continuous days of measurements from $\mathrm{CO}_{2}$ and PIR (passive infrared) motion detectors collected from a collaborative smart space, prior to the start of the pandemic in 2017-2018. We demonstrate how these sensors can be used to estimate occupancy levels, as well as analyze occupancy patterns within the space. We also consider the use of overall air quality within the space for estimating insights about potential transmission risks. Based on our analysis, we derive insights into how infrastructure-based sensors can be used to detect problematic areas in the space and offer guidelines on how to modify these areas to be more social distancing aware.
\end{abstract}

Index Terms - Internet of Things, Social Distancing, COVID19, Occupancy, Air Quality, Indoor Environments, Smart Spaces.

\section{INTRODUCTION}

Social distancing, the use of non-pharmaceutical measures to reduce risk of disease transmission, is a critical tool for mitigating transmission of airborne diseases, such as COVID19. Crowded indoor areas are particularly problematic for disease transmission as viral particles can remain suspended in the air and avoiding contact with other people can be difficult. To assist risk assessment and infection monitoring, wide range of sensing modalities have been proposed as a potential way to understand dynamics of interactions and how they relate to risks of disease transmission [1]. Unfortunately most of the techniques either rely on people carrying the relevant technology (e.g., smartphone-based contact tracing) or on specialized sensing modalities (e.g., magnetic field or thermal imaging) that rarely is easily accessible [2]. Having a widespread and easy-to-understand solution for monitoring social distancing and supporting risk assessment would be essential for safeguarding people residing in indoor spaces and decreasing the risks of disease transmission.

Many smart buildings and spaces incorporate motion and environmental sensors to minimize energy usage. The main purpose for using these solutions is to reduce redundant energy consumption by detecting human presence within the space

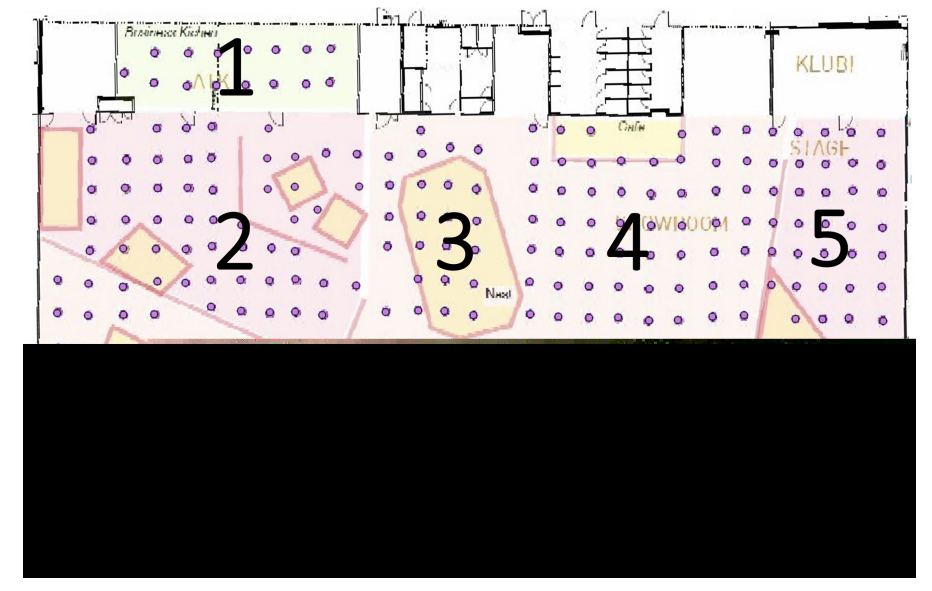

Fig. 1. Tellus smart space.

and to adapt lighting, HVAC, and other energy consuming devices according to human presence, while at the same time ensuring a productive and a healthy environment [3]. As these solutions are already widely deployed, they are an excellent candidate for capturing other types of indoor behaviours.

In this paper, we assess the feasibility of re-purposing the existing infrastructure solutions, specifically sensors for occupancy and indoor air quality monitoring, for the dual purpose of monitoring social distancing and supporting estimation of potential risk factors related to disease transmission. Social interactions are strongly linked with body and other motions, and are good indicators of physical proximity. Environmental sensors, in turn, can potentially support deriving insights about potential risks as current research knowledge suggests that air quality is linked with the transmission of airborne diseases [4], including COVID-19 [5].

We conduct our assessment considering 410 continuous days of measurements from $\mathrm{CO}_{2}$ and PIR (passive infrared) motion detectors collected prior to the start of the pandemic in 2017-2018. The measurements come from the Tellus Innovation Arena $^{1}$ (See Figure 1) at the University of Oulu, Linnanmaa campus [6], which is a collaborative smart learning

${ }^{1}$ https://www.oulu.fi/tellusarena/ 
space. We use the measurements to demonstrate how these sensors can be used to estimate occupancy levels, as well as analyze occupancy patterns within the space. We also consider coarse-grained disease transmission risk estimation using the overall air quality within the space as a proxy for transmission risk. Based on our analysis, we derive insights into how infrastructure-based sensors can be used to detect problematic areas in the space and offer guidelines into how to modify these areas to be more social distancing aware. Tellus is used for teaching and events, has a cafeteria, and open studying spaces, and as such is a representative example of a space that would benefit from crowd avoidance measures, such as those proposed in this paper.

\section{Summary of Contributions:}

1) We demonstrate the potential of repurposing infrastructurebased motion and environmental sensors for occupancy estimation by cross-correlating the motion and $\mathrm{CO}_{2}$ measurements of 352 multisensor devices in a collaborative indoor smart space.

2) We propose a methodology to monitor people density over time, and detect areas that are likely to have less people in the space, and therefore be safer with respect to social distancing.

\section{RELATED WORK}

Internet of Things is gaining significant momentum in supporting healthcare. The COVID-19 pandemic has fueled research on technological means for social distancing to avoid virus infections. Singh et al. [7] conduct a brief review to highlight the significance of IoT applications and propose a roadmap to tackle the pandemic. The study concludes that implementing IoT solutions would reduce healthcare costs as well as improve the treatment of infected patients.

Nasajpour et al. [8] review the role of IoT-based technologies, the architectures, platforms, applications, and industrial IoT-based solutions for combating COVID-19 in three main phases: early diagnosis, quarantine time, and after recovery. This study compares IoT-enabled technologies for the three phases, including wearables, drones, robots, IoT buttons, and smartphone applications for combating COVID-19.

To help with social distancing, Alrashidi et al. [9] aim to find the optimal placement for a set of people equipped with IoT devices to control their locations and movements within an indoor space. This paper uses ant colony optimization and particle swarm optimization for IoT indoor positioning.

Polenta et al. [10] present a system called BubbleBox that combines IoT and software technologies to detect and restrict further outbreaks of the COVID-19 infection through contact tracing. To perform the contact tracing, BubbleBox relies on a dedicated IoT device, a wristband and a web-app that allows users to pair devices with their identities to quickly reach people who might need to be alerted or tested and monitor the spread of the disease. As result, this methodology is seen as an effective approach for monitoring contacts of people who do not own a smartphone such as children or older adults.
Ksentini et al. [11] proposes combining IoT and multiaccess edge computing technologies to build a service that checks and warns people in near real time to help them maintain social distancing. The proposed approach uses a client application that is installed on the user's smartphone, which periodically transmits GPS coordinates to remote servers at the edge of the network. Then, the servers use a social distancing algorithm to warn people who fail to keep sufficient distance.

Hou et al. [12] use deep learning for detecting social distancing by evaluating the distance between people in video frames to limit the transmission of COVID-19. The approach uses pre-trained YOLOv3 object detection model on video frames. Experiments show the proposed method to be effective in monitoring social distancing.

Rusli et al. [13] propose a smart social distancing monitoring system called MySD which stand for "My Safe Distance". MySD leverages smartphone hardware features using Bluetooth transceiver, GPS, Google Maps API and COVID-19 Zone indicator for defining safe distance between people.

In contrast to existing works, we propose monitoring social distancing in smart spaces by re-purposing already deployed infrastructure-based sensors.

\section{DATA COLlection}

\section{A. Tellus Smart Space}

We consider measurements from Tellus smart space at the University of Oulu. The layout of the area is depicted in Figure 1. Tellus includes different smaller spaces such as for meetings rooms, open stage, study, and rest areas. Each Tellus space has its own specific functionality. The Tellus smart space is allocated on the first floor with area size equal to approx $66,856.00 \mathrm{~m} \times 36,446.00 \mathrm{~m}$. The closed spaces that are used for meetings include Galaxy and Horizon which is shown by 1; and rooms Dynamic, Chill, Brisk, and Aspire are shown with numbers 7,8 , and 9 , respectively. The other spaces in the map are used for larger events up to 80 people per space. The Business Kitchen (shown with 2) and Stage space (shown with 5) are used for larger meetings and students' collaborative work. Cafe Tellus (shown with 4) provides coffee and tea facilities; the Nest space (shown by 3 ) is designed for relaxation and thinking. Square which is shown with number 6 is also an open space for students to study individually or within groups. In addition, the yellow cubes indicated with small squares in Figure 1) are tiny closed spaces for up to four people to be used for group meetings [6].

\section{B. Sensors}

The Tellus space uses Low Power Wide Area Network (LPWAN) technology to implement IoT communication. Altogether, 352 LoRa Wide Area Network (LoRaWAN) sensor nodes (Elsys ERS sensors [14]) are deployed into Tellus premises, measuring temperature, humidity, $\mathrm{CO}_{2}$, motion, and light (See Figures 2 and 1). In order to use sensors in field and on the infrastructure, they need to be validated [15]. In our case, the sensors are validated and factory calibrated by the manufacturer prior to deployment. 
Each Elsys ERS sensor node is geo-positioned, providing capabilities for spatial analysis. Each node is powered by two 3.6 V AA lithium batteries and attached to the ceiling frames of the Tellus premises. These sensor nodes are placed in a grid with approximately two-meter spacing between each other, as can be seen from Figure 1. The deployment is described in detail in the original paper [16].

The deployed sensor nodes send data packets every 15 minutes to a remote server utilizing a LoRaWAN radio access network technology. Transmitting on the $868 \mathrm{MHz}$ ISM band, a LoRA gateway manufactured by Multitech is used to collect data from all of the nodes. The gateway is connected to an external biconical D100-1000 antenna from Aerial Oy, and it has an antenna gain of $2 \mathrm{dBi}$. The collected sensor data from this gateway are then transferred using MQTT protocol to the ThingWorx commercial cloud platform, which is then queried by Python scripts to store the data on local servers.

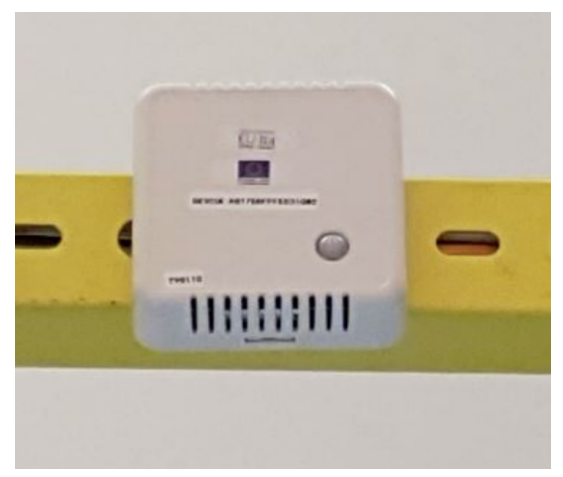

Fig. 2. The multi-sensor device [14].

\section{Dataset}

The data collected from the measurements contains a total of $9,917,848.00$ lines of readings from 352 sensors from 26 June 2017 to 20 November 2018. Each line contains a reading for $\mathrm{CO}_{2}$, humidity, light, temperature and movement (number of movement events within 15 minutes window) for one sensor, identified by an id tag, and a timestamp for when the reading was reported. We also have a mapping that connects 331 of the sensors with their location. The remaining 21 sensors we filter out. Table I summarizes the key statistics of the dataset. The mean and median values of the variables show that the area is often populated $\left(\mathrm{CO}_{2}\right.$ mean, median above 400 , and PIR mean above zero), that it is heated, and that the lights are usually on.

TABLE I

KEY STATISTICS OF THE DATASET.

\begin{tabular}{c|c|c|c|c|c} 
Measurement & Mean & Median & Min & Max & STDEV \\
\hline $\mathrm{CO}_{2}$ & 891.13 & 500.0 & 0.0 & $65,535.00$ & $4,652.73$ \\
Humidity & 30.95 & 31.0 & 0.0 & 100.0 & 11.74 \\
Light & 93.53 & 35.0 & 0.0 & $2,394.00$ & 159.37 \\
Temperature & 21.57 & 21.4 & 0.0 & $6,508.60$ & 7.63 \\
PIR & 2.42 & 0.0 & 0.0 & 144.0 & 4.91
\end{tabular}

\section{Measurements Reliability}

To illustrate the measurement accuracy of sensors deployed in the Tellus space, we selected a random sensor and plotted the measurements of PIR and the $\mathrm{CO}_{2}$. These results are shown in Figure 3. Figure 3(a) presents PIR readings over time of the entire measurement period for the aggregated data to one hour slots. This figure shows that the amount of readings in June - August is lower than other months in 2017 and 2018. On the other hand, the amount of readings in these months 2018 is similar to the amount of readings in the same period in 2017. This reflects the reduced number of students and other occupants of the space during the summer time. In addition, Figure 3(b) shows the readings of the sensor on a randomly selected day. This result explains that with the increasing number of movements under that specific sensor, the amount of $\mathrm{CO}_{2}$ is increasing. In conclusion, these two results state that the multi-device sensor which is capable of detecting the motions and measuring $\mathrm{CO}_{2}$ has been functioning accurately and properly over the measurement period, approving the reliability of the deployed sensors in the space.

\section{DATA ANALYSIS}

\section{A. Correlation of $\mathrm{CO}_{2}$ and Motion Events}

We have shown in previous work [17] that $\mathrm{CO}_{2}$ levels correlate with human activity in an indoor space. We first verify that this holds in the Tellus space.

By using the sensor location maps, we computed at a daily granularity the sum of motion sensor event counts and the mean $\mathrm{CO}_{2}$ level over all sensors. In the dataset, the sensor location mapping file has the MAC address and a running serial number for each sensor. We performed the sensor mapping for the Tellus smart space according to the map in Figure 1. We explored and analyzed the dataset to understand whether the $\mathrm{CO}_{2}$ levels reported by the sensors correlate with the amount of movement in the Tellus space.

We also examined the correlation between activity and $\mathrm{CO}_{2}$ across weekdays. On weekends, the number of visitors is lower, and as a result, both movement events and the mean of $\mathrm{CO}_{2}$ is lower. Figure 4 shows bar plots of detected movement events (subfigure 4(a)) and $\mathrm{CO}_{2}$ concentration (subfigure 4(b)) categorized by day of week. Figure 4(a) shows that there are very few movements detected during the weekend in comparison to the weekdays. This indicates clearly that there are very few people present in the Tellus office on the weekend. The result is in line with the level of $\mathrm{CO}_{2}$ concentration shown in the Figure 4(b). It can be seen that in both weekend days the level of $\mathrm{CO}_{2}$ concentration in the Tellus office are always lower than the weekdays. Computing the sum of motion events for each day in the dataset and grouping the result by weekdays showed that Tuesday is on average the most active day of the week. The day to day variation in the mean $\mathrm{CO}_{2}$ level is less visible, but a clear distinction can be made between weekdays and weekends.

Figure 5 shows scatter plots indicating the relationship between the number of movement ( $\mathrm{x}$-axis, sum of all sensors' 


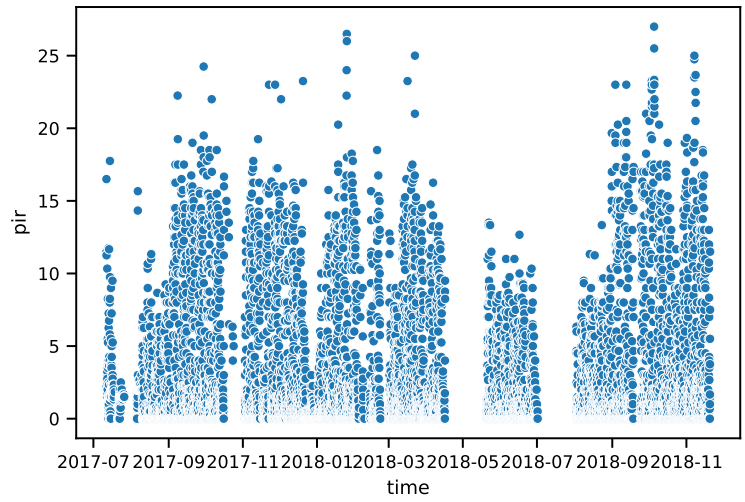

(a) An hour of aggregated PIR (number of movements) measurements over time of the entire measurement period.

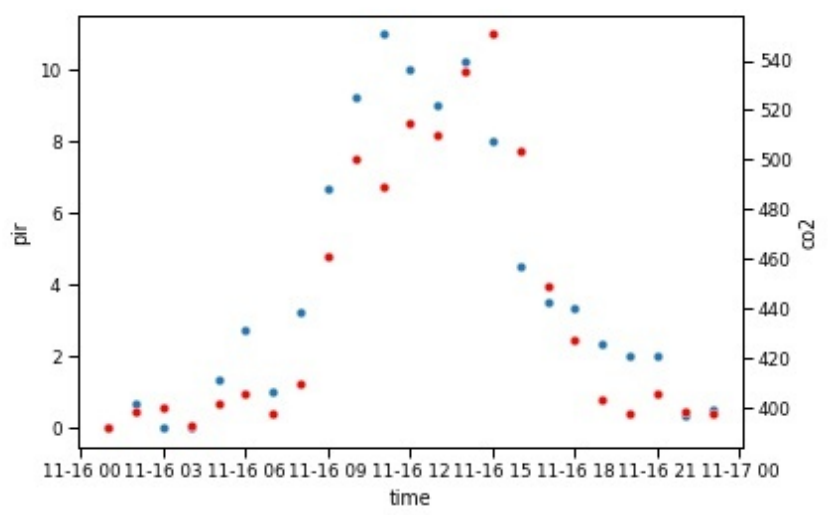

(b) Measurements of a sensor on a day. PIR (number of movements) and $\mathrm{CO}_{2}(\mathrm{ppb})$ measurements are shown with blue and red colors, respectively.

Fig. 3. Sample readings from measurements of a random sensor selected from the space.

movement measurements in a single day) and $\mathrm{CO}_{2}$ concentration (y-axis, mean of all sensors) for weekdays (subfigure 5(a)) and weekends (subfigure 5(b)). We computed the Pearson correlation (R) between these two variables for weekdays and for weekends and holidays. It can be seen that $\mathrm{CO}_{2}$ concentration is strongly associated with the number of people during the weekends $(\mathrm{R}=0.75)$ as well as during the weekdays $(\mathrm{R}=0.57)$. The correlation differences are explained by external factors, such as ventilation systems. During working days, the ventilation systems is continually active and circulates fresh air into the Tellus smart space.

\section{B. Occupancy Estimation}

While motion events and $\mathrm{CO}_{2}$ are correlated, $\mathrm{CO}_{2}$ changes slowly. In this section we estimate the occupancy of the space based on motion events as reported by the sensors in the space.

The mean of motion events of a sensor represents how often that area of the space was occupied in the dataset. Figure 6 shows the overall mean PIR heatmap depicting the locations of the sensors in Tellus smart space. The color denotes the mean

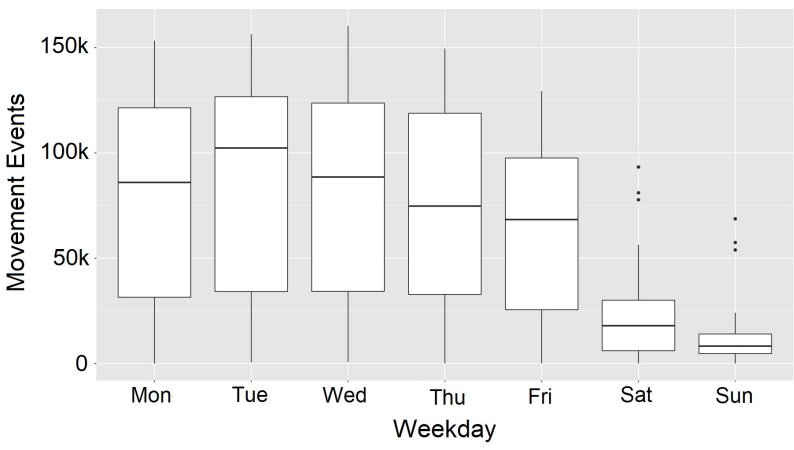

(a) Detected movement events per weekday. Each data point indicates total number of PIR events during the day.

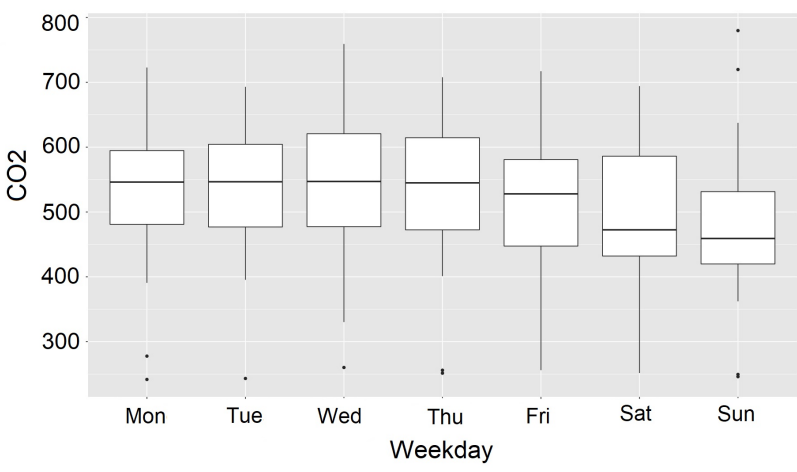

(b) Mean $\mathrm{CO}_{2}(\mathrm{ppb})$ levels per weekday.

Fig. 4. Bar plots of detected movement events and $\mathrm{CO}_{2}(\mathrm{ppb})$ concentration.

motion event count value over all the data points for that sensor in the dataset. The lighter the color, the more movement has been registered overall. The Figure shows a connected line from the cafeteria (shown with 4 in Figure 1) to the open study area (Square, shown with number 6). Sensors near the cafeteria often show occupancy. Otherwise usage is lower and more spread out.

\section{Social Distancing Methodology}

We propose a methodology to monitor people density over time, and detect positions that are likely to have less people in the space, and therefore be safer with respect to social distancing. Based on the results, the space usage can be planned so that different spaces can be used equally. Different spaces can be reserved early. Using the real-time stream of sensor information from each single sensor in the Tellus, the places (even the seats) can be selected with the least number of recorded movements. For example, Figure 6 shows Stage (the area to the top right) is frequently empty, and using it as a study area when no events are planned would reduce the density of people in the study area (bottom left). The area could have portable tables when no events are present.

Since each sensor represents a $2 m \times 2 m$ square in the space, social distancing rules would be easy to follow by staying at the next sensor position or further from each other person in the space. This could be planned by smart placement of work spaces, taking into account small groups of students 


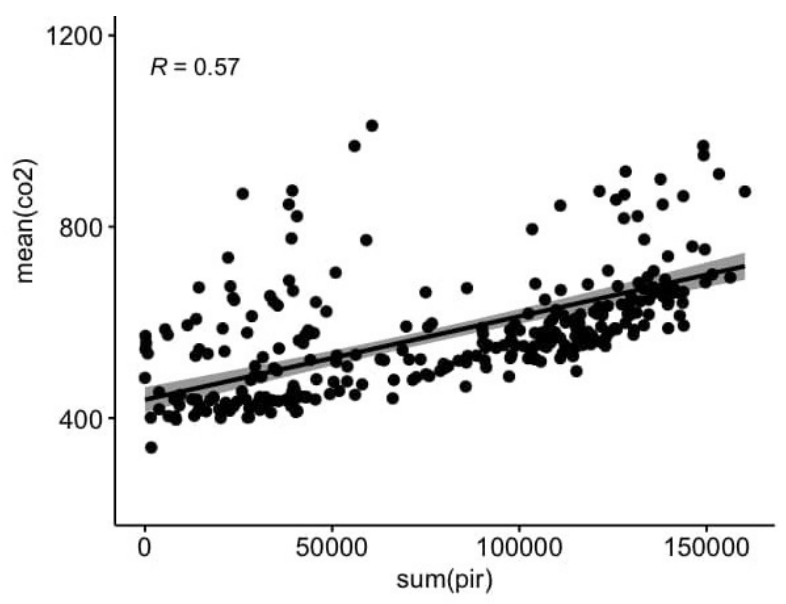

(a) Correlation between motion and $\mathrm{CO}_{2}$ (ppb) during weekdays.

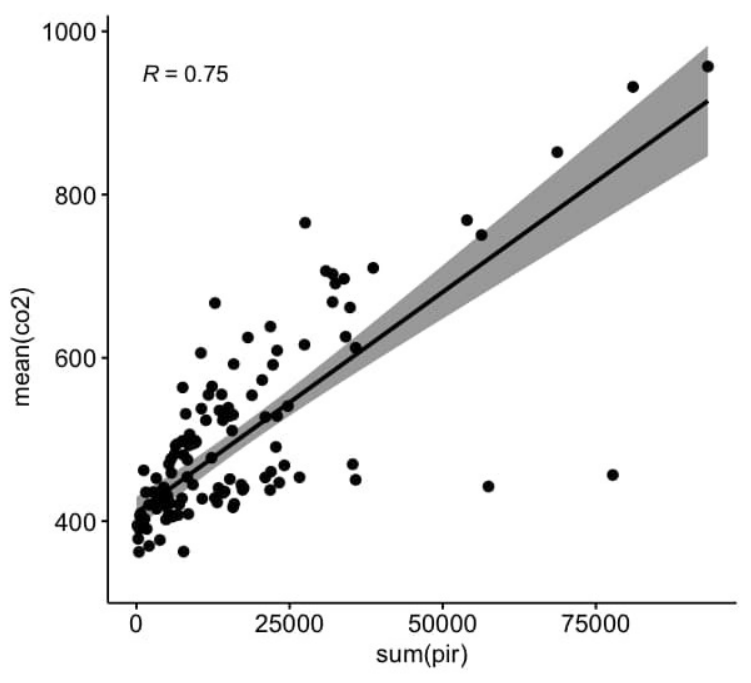

(b) Correlation between motion and $\mathrm{CO}_{2}(\mathrm{ppb})$ during weekends and holidays.

Fig. 5. Scatter plots showing the relationship between the number of movement and $\mathrm{CO}_{2}$ (ppb) concentration.

working together while spacing them further apart from other such workspaces. Sensors could detect the aggregate motion and $\mathrm{CO}_{2}$ over time in such workspaces and estimate the number of users to further keep the number of users below acceptable limits. Upon entering the space, a heatmap of the last hour similar to Figure 6 could be shown to visitors, with directions to the nearest empty space with capacity for a chosen group size.

Once COVID-19 restrictions are eased, the Tellus space will be available for small-scale, fine-grained measurement studies. As less users of the space are allowed inside, it will become possible to obtain reliable ground truth on how the motion and $\mathrm{CO}_{2}$ measurements react to a single person or a small group moving through the space. This will allow us to further validate and fine-tune the methodology.

\section{DISCUSSION}

Applying new approaches for social distancing can not only help prevent virus infections in the spaces, but it may also offer a number of benefits. Followings we present some of possible benefits that may be offered by our methodology for social distancing.

Proxy for social distancing: Since we showed $\mathrm{CO}_{2}$ and motion events are correlated, our methodology can also be applied in spaces with only $\mathrm{CO}_{2}$ sensors. In the other word, the $\mathrm{CO}_{2}$ sensors can be used as proxies for motion detectors [17]. In addition, meeting rooms with $\mathrm{CO}_{2}$ sensors can warn visitors and automatically redirect people to nearby alternatives when the air quality is too low. $\mathrm{CO}_{2}$ readings typically have delayed response which suggests that this could also be used to provide coarse estimates of stay duration.

Virtual sensors: All of smart spaces may not have various air quality sensors for measuring different pollutants. Having one type of sensors measuring $\mathrm{CO}_{2}$ and meteorological variables enables estimating the levels of another key pollutants, such as $\mathrm{PM}_{2.5}$ as described in [18]. The advantage of this approach is on reducing the device, deployment and maintenance costs.

Healthier and comfortable spaces: Social distancing and safe seating areas or working locations can be combined with building automation, e.g., ventilation system can be adjusted to the dedicated spaces and a healthier and comfortable work spaces can be guaranteed [19]. Besides offering healthier environment, this has the added benefit of fostering improved productivity of the space users [20].

Energy and cost savings: By proposing specific seating locations for users, the light levels can be controlled and while ensuring required level of light for the dedicated space, unnecessary lights can be dimmed to lower levels or turned off, hence energy consumption can be reduced [21]. Furthermore, the use of sensors network optimization algorithm enables identifying pollutant hot-spots and finding the optimal numbers of sensors and their locations to be installed. Therefore, the number of sensors to be deployed can be optimized as well as the energy and costs as whole can be reduced significantly.

Improving the space functionality: In long term, the measurements/our methodology shows which places are mostly used and which places are not efficiently used. This enables space and internal designers to plan the better usage of the space and improve the space functionality [22].

Mathematical models: Epidemiological models are important forecasting and preventing virus spreading. Indeed through users density and occupancy detection mathematical models can be defined for social distancing. The space usage can be learnt by the highest number of recorded movements and mostly occupied spaces. Indeed, access to better data and obtaining efficient models enable making proper decisions about the space [23].

Resolution and alternative technologies: The space where our experiments were conducted has dense deployment of PIR sensors while other smart spaces may not have as high resolution. The density of PIR deployments determines the resolution at which social distancing can be monitored. Deployments that are sparser can be used to provide insights about distances even 


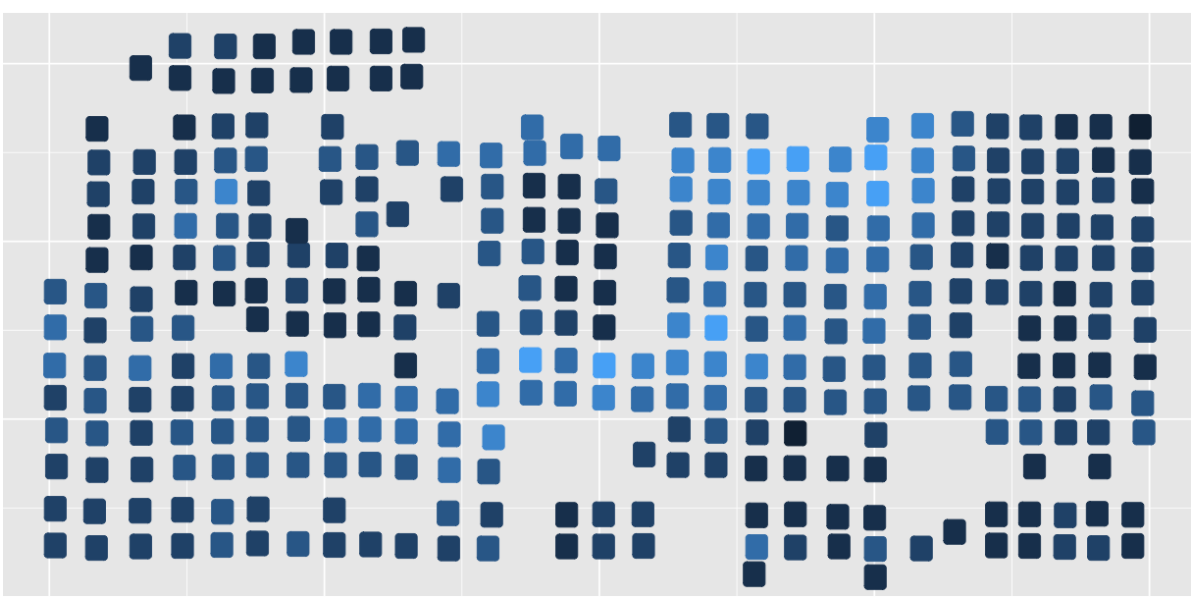

Fig. 6. Overall amount of movement per sensor. The lighter the color, the higher the number of movements.

if they have lower resolution. Alternatively, other technologies can be used to supplement or replace these technologies. For example, low-resolution thermal array sensors are an inexpensive solution for detecting humans, and infrastructure-based WiFi sensing or cameras can equally be used to supplement PIR sensors in monitoring social distancing.

\section{CONCLUSION}

Monitoring and supporting social distancing has become critical for reducing the risks of disease transmission within crowded indoor spaces. In this paper, we used measurements from a collaborative indoor smart space to demonstrate how existing sensor infrastructure, comprising of PIR motion sensors and environmental $\mathrm{CO}_{2}$ sensors, can be repurposed for social distancing monitoring. We also showed how these sensors can be used to identify locations that tend to be crowded and those that are underutilized and safer to occupants. Our work paves the way toward novel infrastructure-based monitoring solutions that can be used to support preventative health countermeasures.

\section{ACKNOWLEDGEMENTS}

This work is supported by Nokia Center for Advanced Research (NCAR), Business Finland (6884/31/2018), Academy of Finland projects with grant numbers 324576 and 335934; and Healthy Outdoor Premises for Everyone (UIA03-240) projects.

\section{REFERENCES}

[1] C. T. Nguyen et al., "A comprehensive survey of enabling and emerging technologies for social distancing-part i: Fundamentals and enabling technologies," IEEE Access, vol. 8, pp. 153 479-153 507, 2020.

[2] C. T. Nguyen, Y. M. Saputra et al., "A comprehensive survey of enabling and emerging technologies for social distancing-part ii: Emerging technologies and open issues," IEEE Access, vol. 8, pp. 154209 $154236,2020$.

[3] N. H. Motlagh et al., "Internet of Things (IoT) and the Energy Sector," Energies, vol. 13, no. 2, p. 494, 2020.

[4] D. Contini and F. Costabile, "Does air pollution influence covid-19 outbreaks?" 2020.

[5] J. L. Domingo et al., "Influence of airborne transmission of sars-cov-2 on covid-19 pandemic. a review," Environmental research, p. 109861, 2020.
[6] E. Gilman et al., "Internet of things for smart spaces: A university campus case study," Sensors, vol. 20, no. 13, p. 3716, 2020.

[7] R. P. Singh et al., "Internet of things (iot) applications to fight against covid-19 pandemic," Diabetes \& Metabolic Syndrome: Clinical Research \& Reviews, 2020.

[8] M. Nasajpour et al., "Internet of things for current covid-19 and future pandemics: An exploratory study," Journal of healthcare informatics research, pp. 1-40, 2020.

[9] M. Alrashidi, "Social distancing in indoor spaces: An intelligent guide based on the internet of things: Covid-19 as a case study," Computers, vol. 9 , no. 4 , p. $91,2020$.

[10] A. Polenta et al., "An internet of things approach to contact tracing- the bubblebox system," Information, vol. 11, no. 7, p. 347, 2020.

[11] A. Ksentini and B. Brik, "An edge-based social distancing detection service to mitigate covid-19 propagation," IEEE Internet of Things Magazine, vol. 3, no. 3, pp. 35-39, 2020.

[12] Y. C. Hou et al., "Social distancing detection with deep learning model," in 2020 8th International Conference on Information Technology and Multimedia (ICIMU). IEEE, 2020, pp. 334-338.

[13] M. E. Rusli et al., "Mysd: A smart social distancing monitoring system," in 2020 8th International Conference on Information Technology and Multimedia (ICIMU). IEEE, 2020, pp. 399-403.

[14] "The Smart Building Sensor for indoor environment monitoring," https: //www.elsys.se/en/ers/, accessed: 2021-01-15.

[15] N. H. Motlagh et al., "Low-cost air quality sensing process: Validation by indoor-outdoor measurements," in 15th IEEE Conference on Industrial Electronics and Applications (ICIEA2020). IEEE, 2020.

[16] R. Yasmin et al., "Combining iot deployment and data visualization: experiences within campus maintenance use-case," in 2018 9th International Conference on the Network of the Future (NOF). IEEE, 2018, pp. 101-105.

[17] N. H. Motlagh et al., "Indoor air quality monitoring using infrastructurebased motion detectors," in 2019 IEEE 17th International Conference on Industrial Informatics (INDIN), vol. 1. IEEE, 2019, pp. 902-907.

[18] M. A. Zaidan et al., "Intelligent calibration and virtual sensing for integrated low-cost air quality sensors," IEEE Sensors Journal, vol. 20, no. 22 , pp. $13638-13652,2020$.

[19] H. Chappells, "Comfort, well-being and the socio-technical dynamics of everyday life," Intelligent Buildings International, vol. 2, no. 4, pp. 286-298, 2010.

[20] S. Tanabe et al., "Workplace productivity and individual thermal satisfaction," Building and environment, vol. 91, pp. 42-50, 2015.

[21] N. H. Motlagh et al., "Toward sustainable energy-independent buildings using internet of things," Energies, vol. 13, no. 22, p. 5954, 2020.

[22] F. Cassol et al., "Multi-objective optimization as a new approach to illumination design of interior spaces," Building and Environment, vol. 46, no. 2, pp. 331-338, 2011.

[23] Microsoft. AI for Health. [Online]. Available: https://www.microsoft. com/en-us/ai/ai-for-health/ 